\title{
Potentially active microbes in the sediment of hadal trenches and their metabolic potentials: implications to organic carbon degaradation in hadal sediments
}

Rulong LiU ${ }^{1 *}$ Li WaNG ${ }^{1}$, ZiXUAN WANG ${ }^{1}$, XING WEI $^{1}$, WEIZHI SONG ${ }^{2}$, JIASONG FANG ${ }^{1,3}$

${ }^{1}$ College of Marine Science, Shanghai Ocean University, Shanghai, China

${ }^{2}$ Centre for Marine Bio-Innovation, University of New South Wales, Sydney, Australia

${ }^{3}$ Qingdao National Laboratory for Marine Science and Technology, Qingdao, China

Surprisingly high rates of microbial carbon turnover have been recently reported in the sediment of several hadal trenches, making the hadal zone "hot spots" for organic carbon degradation in the deep ocean. However, the potentially active microbial taxa and their metabolic mechanism that dictate the biodegradation of organic carbon in the hadal zone are largely unknown. We studied the community composition and spatial variations of potentially active microbial community in $1 \mathrm{~cm}$ sectioned surface sediments (full depth $=10 \mathrm{~cm}$ ) from the deepest points of the Mariana and Mussau trenches, and the interactions between different active taxa were also explored using co-occurrence network. The micbial lineages with the highest activity potentials or those playing keystone roles in co-occurrence network were identified as important candidatus taxa in driving organic carbon degradation. The metabolic potentials and organic carbon degradation pathways of those important taxa were analyzed, based on metagenomic assembled genomes. The results showed that those important taxa contained pathways for degradation of diverse organic carbon substrates, including several types of recalcitrant organic matter that may present in the hadal sediments, such as alkane, lignin. The study provide valuable insights to the microbial mecahnismes that are driving the active organic carbon degradation in hadal sediments. 